\title{
La variole du dromadaire chez le jeune au Maroc. Isolement et identification du virus. Mise au point du vaccin et application à la prophylaxie
}

\author{
M. El Harrak ${ }^{1}$ C. Loutfi ${ }^{1}$
}

\section{Mots-clés}

Dromadaire - Jeune animal - Orthopoxvirus - Vaccin - Contrôle des maladies - Maroc.

\begin{abstract}
Résumé
La variole cameline est une pathologie majeure de l'espèce. Le virus (O rthopoxvirus cameli) a pu être isolé au Maroc. La souche isolée après huit passages sur cellules Vero a été dénommée T8. Un vaccin inactivé a pu être mis au point et évalué par le test de séroneutralisation et par la détermination de l'index de protection après l'épreuve virulente. Ce vaccin a été utilisé dans les campagnes de vaccination au Maroc à partir de 1993. Chaque année, 15000 jeunes dromadaires sont immunisés. Son innocuité est totale et son efficacité reconnue.
\end{abstract}

\section{INTRO DUCTION}

La variole du dromadaire (ou camel pox) est l'une des maladies infectieuses du dromadaire les plus propagées dans le monde. Elle est due à un orthopoxvirus (Orthopoxvirus cameli) et se manifeste par l'apparition de lésions éruptives ayant une allure papulopustuleuse à localisations multiples avec une fréquence constante sur la face et les extrémités distales des membres.

Chez le jeune de 1 à 3 ans, la maladie revêt un caractère de gravité médicale plus accentué et elle peut engendrer la mort. Dans la forme grave, la généralisation se fait en 8 à 15 jours avec atteinte des muqueuses externes et même internes. Les chamelons de moins de six mois ne contractent pas la maladie, ils bénéficient encore de l'immunité passive.

$\mathrm{Au}$ Maroc la maladie est connue depuis longtemps et est redoutée des éleveurs sous le nom de jedri. Elle sévit annuellement dans les régions du sud du pays où une morbidité de 100 p. 100 et un taux de mortalité de 20 à 30 p. 100 chez les jeunes entraînent des pertes économiques considérables. Son étiologie n'a été confirmée qu'au cours d'une épizootie survenue dans le Sud pendant l'été 1984. Les premiers cas ont été déclarés à l'Est près de la région d'Ouarzazate, avant de se propager aux provinces du Sud. L'apparition de cette épidémie semble être en relation avec trois facteurs essentiels :

1. Biopharma, Département de virologie, BP 4569, Akkari, Rabat, Maroc
- le repeuplement des régions sahariennes en dromadaires ;

- la multiplication des déplacements des troupeaux en quête de pâturages (sécheresse) ;

- la grande sensibilité des jeunes immunologiquement vierges.

La mise en place d'une prophylaxie médicale s'est avérée nécessaire pour éviter la propagation de la maladie dans les zones indemnes. Un vaccin inactivé adjuvé a alors été mis au point à partir de la souche locale et a été utilisé depuis 1991.

En 1993, une nouvelle éclosion de la maladie s'est déclarée dans les provinces du Sud et de nouveaux foyers ont été signalés. Cependant la maladie a été rapidement contrôlée par l'instauration de mesures de prophylaxie sanitaire dans les zones infectées et la vaccination des dromadaires autour des foyers. La généralisation de la vaccination dans toutes les provinces du Sud a été par la suite appliquée avec un rappel vaccinal annuel des jeunes dromadaires.

\section{ISO LEM EN T ET IDEN TIFICATIO N}

DU VIRUS DE LA VARIOLE

DU DROMADAIRE AU MAROC

Le virus, isolé à partir de croûtes sèches de jeunes dromadaires pendant l'épizootie de 1984, a été passé sur la membrane chorioallantoïdienne d'embryon de poulet puis adapté à la lignée cellulaire Vero. Le virus a été dénommé T8 à son $8^{e}$ passage sur cellules. 
L'effet cytopathogène de la souche T8 s'est caractérisé par l'apparition d'inclusions intracytoplasmiques et par la formation de gros syncytia ronds avec des noyaux disposés en couronne dont la nécrose a donné des plages de lyse rondes qui, après confluence, ont entraîné la destruction du tapis cellulaire (figure 1). Le virus cultivé sur cellules Vero a montré un titre de 5,5 log DICT 50 par millilitre à $37^{\circ} \mathrm{C}$ (tableau I).

La souche T8 a été très sensible aux solvants lipidiques (éther et chloroforme). Le traitement par une solution de trypsine à 2,5 p. 100 pendant 15 min à $37^{\circ} \mathrm{C}$ a fait diminuer le titre viral de $1,2 \mathrm{log}$. Le virus s'est également avéré sensible à la chaleur ; il s'est complètement inactivé après 15 min à $56^{\circ} \mathrm{C}$ (figure 2 ).

Une étude des caractères ultrastructuraux a été réalisée par microscopie électronique (figure 3). Elle a montré que le virus a suivi un cycle normal d'adsorption, de pénétration, de décapsidation puis de synthèse du matériel viral. Les particules virales définitives sont restées liées à la cellule, le bourgeonnement n'a pas été observé.

\section{MISE AU POINT DU VACCIN INACTIVE}

La souche T8 a été utilisée pour la production du vaccin inactivé. Le virus a été cultivé sur les cellules Vero dans des flacons roulants. La suspension virale récoltée après généralisation de l'effet cythopathique a été inactivée avec le formol puis absorbée sur l'hydroxyde d'alumine.

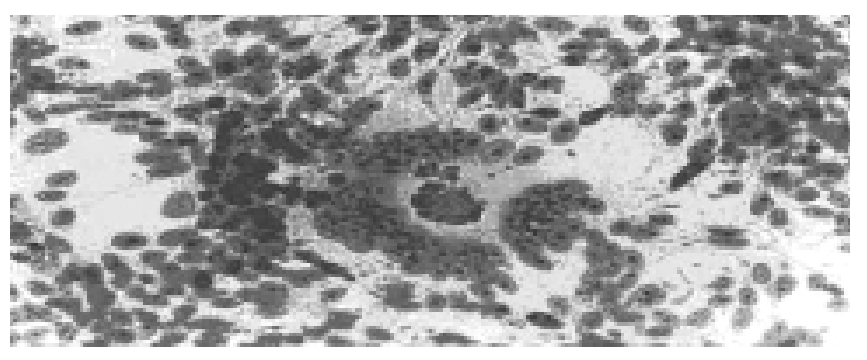

Figure 1 : cellules Vero infectées avec la souche T8; syncytia ronds (x 100); coloration MGG.

Tableau I

Titre viral en fonction de la température d'incubation

\begin{tabular}{lccc}
$\begin{array}{l}\text { Température } \\
\text { d'incubation } \\
\left({ }^{\circ} \mathbf{C}\right)\end{array}$ & \multicolumn{3}{c}{ Titre infectieux log DICT $\mathbf{5 0 / \mathbf { m l }}$} \\
\cline { 2 - 4 } & $\begin{array}{l}\text { Virus } \\
\text { total }\end{array}$ & $\begin{array}{c}\text { Virus } \\
\text { intracellulaire }\end{array}$ & $\begin{array}{c}\text { Virus } \\
\text { extracellulaire }\end{array}$ \\
\hline 28 & 6,3 & 6,2 & 4,4 \\
30 & 6,4 & 6,2 & 4,4 \\
33 & 6,3 & 6,3 & 4,2 \\
35 & 6,5 & nd & nd \\
37 & 5,5 & 6,2 & 2,5 \\
38 & 4,0 & nd & nd \\
\hline
\end{tabular}

nd = non déterminé
Le contrôle d'innocuité a été réalisé sur quatre chamelons primovaccinés par voie sous-cutanée avec une dose vaccinale de $5 \mathrm{ml}$ renfermant 6,8 log DECP 50 du virus vaccinal. L'innocuité spécifique a été établie par l'absence de la généralisation de la maladie ou d'une réaction locale importante au cours des 21 jours qui ont suivi l'inoculation. Les réponses sérologiques et immunologiques des animaux vaccinés ont été évaluées par le test de séroneutralisation et par la détermination de l'index de protection après l'épreuve virulente.

Les résultats obtenus montrent une innocuité parfaite, aucune réaction postvaccinale ou élévation thermique n'a été enregistrée après la vaccination. Une bonne réponse sérologique a été détectée à partir du $7^{\mathrm{e}}$ jour, ainsi qu'un pouvoir protecteur satisfaisant avec un indice de protection moyen de 2,5 log.

\section{CONTRO LE SUR LE TERRAIN DU VACCIN INACTIVE ET APPLICATION A LA PROPHYLAXIE AU MAROC}

La prophylaxie de la variole du dromadaire a été conduite au Maroc et dans d'autres pays grâce au vaccin inactivé depuis 1991 (figure 4). Avant son utilisation à grande échelle, ce vaccin a subi un essai clinique sur le terrain en vaccinant un troupeau contrôlé de jeunes, d'adultes et de femelles gestantes. Le suivi de ce troupeau a permis de constater l'innocuité totale du vaccin et sa capacité d'induire une protection révélée par le taux des anticorps neutralisants (figure 5).

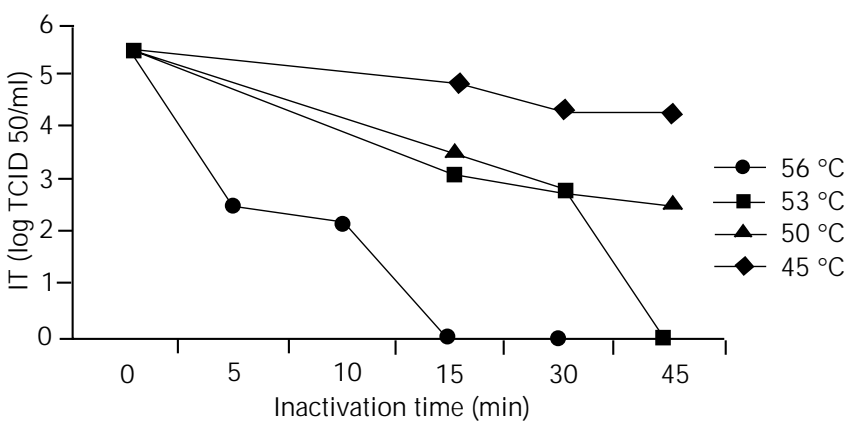

Figure 2 : inactivation de la souche T8 par la température.

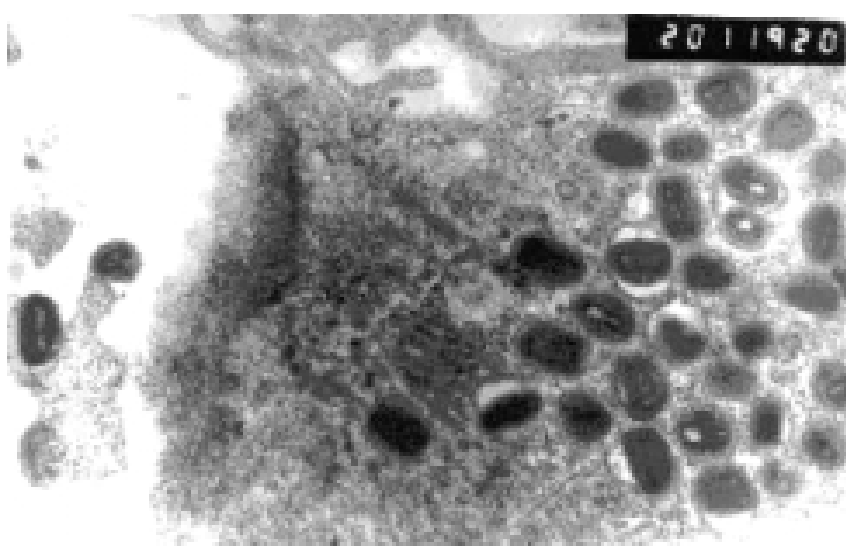

Figure 3 : cellules Vero infectées par la souche T8; particules virales matures (x 40 000). 


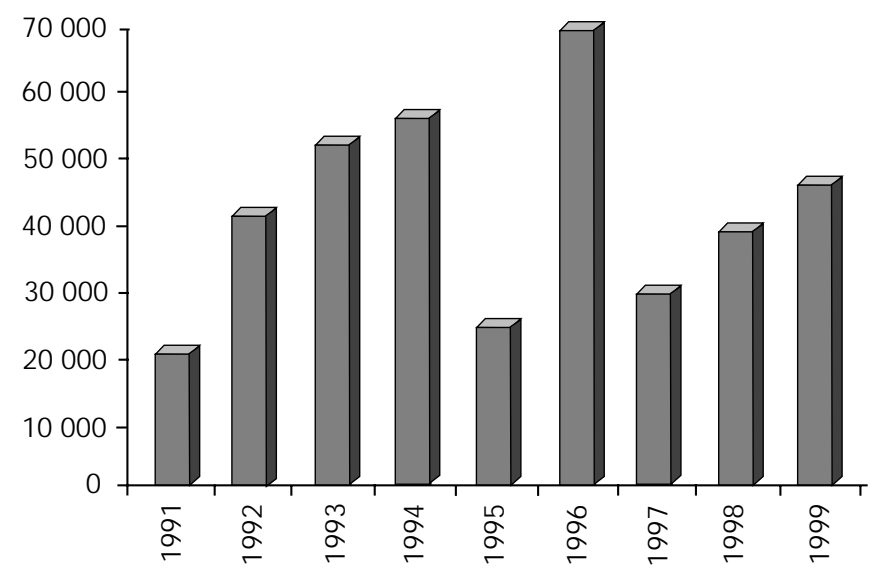

Figure 4 : production du vaccin CPV inactivé au Maroc. Le taux des productions varie selon les commandes formulées par les pays utilisateurs. Plus de la moitié de la production est exportée principalement vers les pays de l'Afrique du Nord.

Ce vaccin a été utilisé dans les campagnes de vaccination annuelles organisées par la Direction de l'élevage au Maroc. A partir de 1993, des campagnes de prophylaxie de masse ont été réalisées dans toutes les provinces du Sud marocain.

Les effectifs vaccinés ont varié d'une année à l'autre (entre 7000 et 22000 ) selon l'incidence de la maladie. Entre 1993 et 1997, 15000 jeunes dromadaires en moyenne par an ont été immunisés.

La situation sanitaire actuelle de la maladie est jugée satisfaisante, mais des enquêtes sérologiques en cours ont montré la présence d'environ 50 p. 100 d'animaux séronégatifs dans le cheptel marocain. Une couverture vaccinale plus élevée et atteignant un

\section{Summary}

El Harrak M., Loutfi C. Camel pox in the calf in Morocco. Identification of the isolated virus. Development and application to prophylaxis

Camel pox is a major disease of camels. The virus (O rthopoxvirus cameli) was isolated in Morocco after eight passages of the strain on Vero cells; it was named T8. An inactivated vaccine was developed and assessed by the seroneutralization test and determination of the protection index after challenge. Starting in 1993 it was used in vaccination campaigns in M orocco. Every year 15,000 young camels are immunized. It is totally innocuous and its efficacy is acknowledged.

Key words: Dromedary - Young animal - Orthopoxvirus Vaccine - Disease control - Morocco.

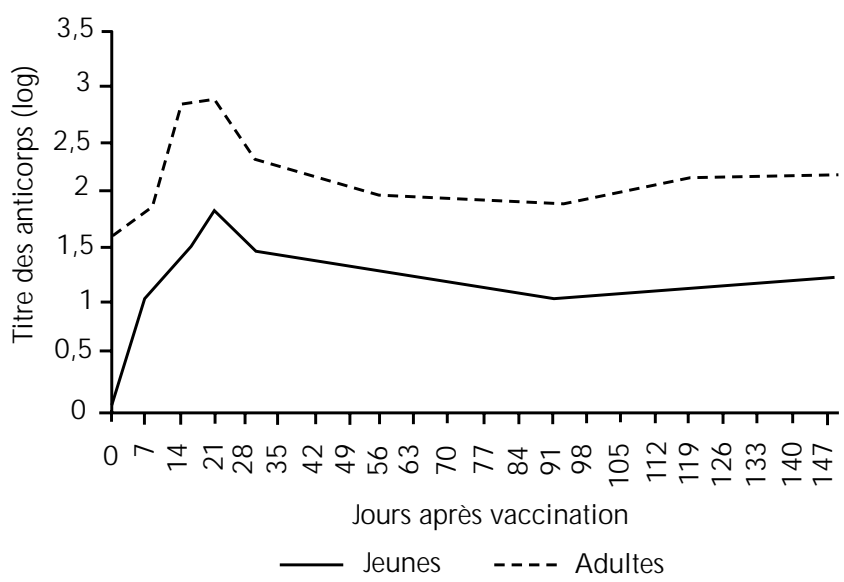

Figure 5 : résultats sérologiques du contrôle sur le terrain du vaccin inactivé.

minimum de 80 p. 100 des jeunes serait souhaitable pour prévenir toute résurgence de la maladie.

\section{CONCLUSION}

Lors d'une épizootie survenue en 1984, un virus de la famille des orthopoxvirus a été isolé et identifié comme l'agent responsable de la variole du dromadaire dans le Sud marocain. L'adaptation à la culture sur lignées cellulaires a été réussie et un vaccin inactivé adjuvé a été mis au point. La vaccination de masse est conduite au Maroc depuis 1993 grâce au vaccin inactivé qui a fait preuve d'innocuité totale et d'efficacité. Ce vaccin a été également exporté dans d'autres pays.

\section{Resumen}

El Harrak M., Loutfi C. La viruela del dromedario en el joven en Marruecos. Aislamiento e identificación del virus. Desarrollo de una vacuna y aplicación a la profilaxis

La viruela camélida es una patología mayor de la especie. El virus (O rthopoxvirus cameli) ha sido aislado en Marruecos. La cepa, aislada después de ocho pasajes en células Vero, se denominó T8. Se logró desarrollar una vacuna inactivada, la cual se evaluó mediante el test de neutralización sérica, así como por la determinación del índice de protección después de la prueba virulenta. Esta vacuna se utilizó en las campañas de vacunación en Marruecos a partir de 1993. Cada año, 15000 jóvenes dromedarios son inmunizados. Su inocuidad es total y su eficiencia reconocida.

Palabras clave: Dromedario - Animal joven - Orthopoxvirus Vacuna - Control de enfermedad - M arruecos. 